\title{
ON THE HOMOLOGY OF FINITE CYCLIC COVERINGS OF HIGHER-DIMENSIONAL LINKS
}

\author{
D. W. SUMNERS ${ }^{1}$
}

ABSTRACT. We produce an explicit formula for the betti numbers of the $k$-fold branched cyclic covering of a link, in terms of complex $k$ th roots of unity which are also roots of the polynomial invariants of the link. More information is obtained when $k$ is a prime power.

I. Introduction. For classical knots and links, there is a well-known formula [2], [3], [8] for computing the 1-dimensional betti numbers of the $k$-fold branched cyclic covering space. The formula is given in terms of complex $k$ th roots of unity which are also roots of the polynomial invariants of the infinite cyclic covering space of the complement. This paper gives a new proof of these results, and extends them to a calculation of all the betti numbers for a higher-dimensional link. The proof exploits a long exact sequence [5] relating the homology of the infinite cyclic cover to that of the $k$-fold unbranched cyclic cover.

The formulas are particularly simple and interesting in the case of prime power coverings (see Theorem 3 and Corollary 5). Moreover, the method of proof of Theorem 3 has turned out to be useful in the study of the monodromy of plane algebraic curves [7].

II. Rational homology invariants. An $n$-link of multiplicity $\mu$ denotes a smooth oriented submanifold $L_{\mu}^{n}$ of $S^{n+2}$ homeomorphic to $\mu$ disjoint copies of $S^{n}$. If $X$ denotes the complement of the link, then A lexander duality gives us

$$
\begin{aligned}
H_{i}(X ; Z) & =\mu Z, & & i=1, \\
& =(\mu-1) Z, & & i=n+1, \\
& =Z, & & i=0, \\
& =0, & & \text { otherwise, }
\end{aligned}
$$

Received by the editors September 24, 1973.

AMS (MOS) subject classifications (1970). Primary 57C45, 55A10, 55A25.

Key words and phrases. Higher-dimensional link, infinite cyclic cover, $k$-fold unbranched cyclic cover, branched cyclic cover.

1 Research partially supported by NSF GP-19964. 
where $\alpha Z$ denotes the direct sum of $\alpha$ copies of $Z$.

The orientation of the manifold pair $\left(S^{n+2}, L_{\mu}^{n}\right)$ allows us to define the homological linking number $\left\langle\tau, L_{\mu}^{n}\right\rangle$ between 1 -cycles $\tau$ and the link $L_{\mu}^{n}$. This determines a homomorphism

$$
\pi_{1}(X) \stackrel{\phi}{\rightarrow} J(t), \quad \tau \mapsto t^{\left\langle\tau, L_{\mu}^{n}\right\rangle}
$$

where $J(t)$ is the infinite cyclic multiplicative group on the generator $t$. The infinite cyclic covering space $\tilde{X}$ of $X$ is the regular covering space associated with $\operatorname{Ker} \phi . \tilde{X}$ is an invariant of oriented link type.

Let $Z, Q$ and $C$ denote the integers, rationals and complex numbers. Let $\Lambda, \Gamma$ and $\Psi$ denote the integral, rational and complex group ring of $J(t)$, respectively. We have $\Gamma \cong \Lambda \otimes_{z} Q$ and $\Psi \cong \Gamma \otimes_{Q} C$ and regard $\Lambda \subset \Gamma$ $\subset \Psi$ as subrings. $\Lambda$ is a Noetherian unique factorization domain, and $\Gamma$ and $\Psi$ are principal ideal domains. Since $J(t)$ acts on $\tilde{X}$ as the group of covering translations, we have that $H_{q}(\tilde{X} ; Z)$ is a finitely-presented $\Lambda$-module for all $q$, and likewise for $H_{q}(\tilde{X} ; Q)$ and $H_{q}(\tilde{X} ; C)$ over $\Gamma$ and $\Psi$. The polynomial invariants are the invariants of the $\Gamma$-structure of $H_{q}(\widetilde{X} ; Q)$. Since $\Gamma$ is a $\mathrm{PID}$, we have that $H_{q}(\tilde{X} ; Q) \cong_{\Gamma} F_{q} \oplus T_{q}$ where $F_{q}$ is $\Gamma$-free and $T_{q}$ is $\Gamma$-torsion. If $\lambda \in \Gamma$, let $\Gamma / \lambda$ denote the cokernel of multiplication by $\lambda$ on $\Gamma$. It is shown in [5] that in the range $n \geq 2$ or $n=1, \mu=1$ that

$$
\begin{aligned}
F_{q} & =(\mu-1) \Gamma, & & q=1, n+1, \\
& =0, & & \text { otherwise }
\end{aligned}
$$

and $T_{q} \cong \Gamma / \lambda_{1}^{q} \oplus \cdots \oplus \Gamma / \lambda_{m_{q}}^{q}$ where $\lambda_{i}^{q} \in \Lambda, \lambda_{i+1}^{q} \mid \lambda_{i}^{q}$ in $\Lambda, 1 \leq i \leq\left(m_{q}-1\right)$. Moreover, if $q \geq 1$ then $\lambda_{i}^{q}(1)= \pm 1 \forall i$; and $\lambda_{1}^{0}(t)=(t-1)$. The rank of $F_{q}$ together with the invariant factors $\left\{\lambda_{i}^{q}\right\}$ are a complete set of invariants for the $\Gamma$-module $H_{q}(\tilde{X} ; Q)$, hence are invariants of oriented link type.

Moreover, the $\Gamma$-structure of $H_{q}(\widetilde{X} ; Q)$ determines the $\Psi$-structure of $H_{q}(\widetilde{X} ; C) \cong H_{q}(\widetilde{X} ; Q) \otimes_{Q} C$. That is, for $1 \leq q \leq n$, the $\Psi$-torsion summand of $H_{q}(\tilde{X} ; C)$ is $\Psi / \lambda_{i}^{q} \oplus \cdots \oplus \Psi / \lambda_{m_{q}}^{q}$, and the $\bar{\Psi}_{\text {-rank of }}$

$$
\begin{aligned}
H_{q}(\tilde{X} ; C) & =(\mu-1), & & q=1, n+1, \\
& =0, & & \text { otherwise. }
\end{aligned}
$$

The $k$-fold unbranched cyclic cover $X_{k}^{u}$ of $X$ is the regular covering associated with the canonical epimorphism 


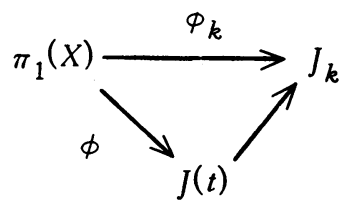

where $J_{k}$ denotes the finite cyclic multiplicative group of order $k$. The unbranched cover has a unique completion, the associated $k$-fold branched cyclic cover $X_{k}^{b}$, branched along the link $L_{\mu}^{n}$. Let $k$ be fixed, and let $\alpha_{i}^{q}$ be the number of distinct $k$ th roots of unity which are roots of $\lambda_{i}^{q}$. Let $c_{q}=$ $\sum_{i=1}^{m} \alpha_{i}^{q}$, and let $\beta_{q}=q$-dimensional betti number of $X_{k}^{u}$. We have the following

Theorem 1. $n \geq 2$ or $n=1, \mu=1$. Then

$$
\begin{aligned}
\beta_{q} & =c_{q}+c_{q-1}, & & 2 \leq q \leq n, \\
& =k(\mu-1)+c_{1}+1, & & q=1, \\
& =k(\mu-1)+c_{n}, & & q=n+1, \\
& =1, & & q=0 .
\end{aligned}
$$

Proof. We have that $\beta_{q}=\operatorname{rank}_{Q}\left(H_{q}\left(X_{k}^{u} ; Q\right)\right)=\operatorname{rank}_{C}\left(H_{q}\left(X_{k}^{u} ; C\right)\right)$, so it will suffice to compute the complex rank. As in [5], we have the short exact sequence of chain complexes (over $\Psi$ and $C$ ):

$$
0 \rightarrow C_{*}(\tilde{X} ; C) \stackrel{\left(t^{k}-1\right)}{\longrightarrow} C_{*}(\tilde{X} ; C) \rightarrow C_{*}\left(X_{k}^{u} ; C\right) \rightarrow 0
$$

This induces the long exact sequence of homology

$$
\cdots \rightarrow H_{q}(\tilde{X} ; C) \stackrel{\left(t^{k}-1\right)}{\longrightarrow} H_{q}(\tilde{X} ; C) \rightarrow H_{q}\left(X_{k}^{u} ; C\right) \stackrel{\partial}{\rightarrow} \cdots
$$

Let $\mathrm{Ker}_{q}$ and $\mathrm{Cok}_{q}$ denote the kernel and cokernel, respectively, of the map $\left(t^{k}-1\right)$; then the following is an exact sequence of $C$-vector spaces:

$$
0 \rightarrow \operatorname{Ker}_{q} \rightarrow H_{q}(\tilde{X} ; C) \stackrel{\left(t^{k}-1\right)}{\longrightarrow} H_{q}(\tilde{X} ; C) \rightarrow \operatorname{Cok}_{q} \rightarrow 0
$$

Note that in the range $2 \leq q \leq n, H_{q}(\tilde{X} ; C)$ is pure $\Psi$-torsion, so is a finitedimensional $C$-vector space, and hence $\operatorname{dim}_{C}\left(\operatorname{Ker}_{q}\right)=\operatorname{dim}_{C}\left(\operatorname{Cok}_{q}\right)$. The long exact sequence (1) decomposes into a number of short exact sequences

$$
0 \rightarrow \operatorname{Cok}_{q} \rightarrow H_{q}\left(X_{k}^{u} ; C\right) \rightarrow \operatorname{Ker}_{q-1} \rightarrow 0
$$


So $\operatorname{dim}_{C} H_{q}\left(X_{k}^{u} ; C\right)=\operatorname{dim}_{C} \operatorname{Ker}_{q-1}+\operatorname{dim}_{C} \operatorname{Cok}_{q}$.

Note that the homomorphism $\left(t^{k}-1\right)$ respects any $\Psi$-splitting for $H_{q}(\tilde{X} ; C)$. It will therefore suffice in most instances to calculate the kernel and cokernel of $\left(t^{k}-1\right)$ restricted to a single cyclic summand of the form $\Psi / \lambda$. $\lambda$ has a prime factorization in $\Psi$ into powers of linear factors $\lambda=$ $\Pi_{j}\left(t-z_{j}\right)^{e}{ }_{j}$. We can further decompose $\Psi / \lambda$ into the direct sum

$$
\Psi /\left(t-z_{1}\right)^{e} 1 \oplus \ldots
$$

so focus attention on a prime-power summand $\Psi /\left(t-z_{j}\right)^{e} j$. Since $\left(t^{k}-1\right)=$ $\Pi_{i=0}^{k-1}\left(t-\zeta^{i}\right)$ where $\zeta$ is a primitive $k$ th root of unity, we have the exact sequence

$$
0 \rightarrow \operatorname{Ker} \rightarrow \Psi /\left(t-z_{j}\right)^{e_{j}} \stackrel{\left(t^{k}-1\right)}{\longrightarrow} \Psi /\left(t-z_{j}\right)^{e_{j}} \rightarrow \text { Cok } \rightarrow 0
$$

where

$$
\begin{aligned}
\mathrm{Ker} \cong \mathrm{Cok} & \cong C \quad \text { if } z_{j} \text { is a } k \text { th root of unity } \\
& \cong 0 \quad \text { otherwise }
\end{aligned}
$$

Hence the theorem is proved in the range $3 \leq q \leq n$. Since

$$
H_{n+2}(\tilde{X} ; C) \cong(\mu-1) \Psi \quad \text { and } \quad 0 \rightarrow \Psi \stackrel{\left(t^{k}-1\right)}{\longrightarrow} \Psi-k C-0
$$

is exact, then $\operatorname{Cok}_{n+1} \cong k(\mu-1) C$. So rank ${ }_{C} H_{n+1}\left(X_{k}^{u} ; C\right)=k(\mu-1)+c_{n}$. Likewise $\mathrm{Ker}_{1}$ gets contributions only from the $\stackrel{n}{\Psi}$-torsion part of $H_{1}(\widetilde{X} ; \stackrel{n}{C})$ so rank $\mathrm{H}_{2}\left(X_{k}^{u} ; C\right)=c_{2}+c_{1}$. In dimension 1 , we have the following exact sequence:

$$
0 \rightarrow\left[k(\mu-1)+c_{1}\right] C \rightarrow H_{1}\left(X_{k}^{u} ; C\right) \rightarrow H_{0}(\tilde{X} ; C) \stackrel{0}{\rightarrow} H_{0}(\tilde{X} ; C) \rightarrow \cdots
$$

Hence rank ${ }_{C} H_{1}\left(X_{k}^{u} ; C\right)=k(\mu-1)+c_{1}+1$. This completes the proof of Theorem 1.

Corollary 2. Let $X_{k}^{b}$ be the $k$-fold branched cyclic cover of $X$, and $n \geq 2$ or $n=1, \mu=1$, and $\bar{\beta}_{q}=q$-dimensional betti number of $X_{k}^{b}$. Then

$$
\begin{aligned}
\beta_{q} & =c_{q}+c_{q-1}, & & 2 \leq q \leq n, \\
& =(k-1)(\mu-1)+c_{1}, & & q=1, \\
& =(k-1)(\mu-1)+c_{n}, & & q=n+1, \\
& =1, & & q=0, n+2 .
\end{aligned}
$$


Proof. By excision,

$$
\begin{aligned}
H_{i}\left(X_{k}^{b}, X_{k}^{u} ; C\right) & =\mu C, & & i=2, n+2, \\
& =0, & & \text { otherwise. }
\end{aligned}
$$

Just as in [5], we have that $X_{k}^{b}$ is orientable, and the exact sequence of the pair $\left(X_{k}^{b}, X_{k}^{u}\right)$ yields

$$
0 \rightarrow H_{n+2}\left(X_{k}^{b}\right) \stackrel{j_{*}}{\rightarrow} H_{n+2}\left(X_{k}^{b}, X_{k}^{u}\right) \rightarrow H_{n+1}\left(X_{k}^{u}\right) \rightarrow H_{n+1}\left(X_{k}^{b}\right) \rightarrow 0 .
$$

Moreover, $j_{*}$ takes the orientation class onto the element $(1,1, \ldots, 1)$ in $C \oplus \cdots \oplus C \cong H_{n+2}\left(X_{k}^{b} ; X_{k}^{u}\right)$, so we have that

$$
\begin{aligned}
\operatorname{rank}_{C} H_{n+1}\left(X_{k}^{b} ; C\right) & =\operatorname{rank}_{C} H_{n+1}\left(X_{k}^{u} ; C\right)-(\mu-1) \\
& =k(\mu-1)+c_{n}-(\mu-1)=(k-1)(\mu-1)+c_{n} .
\end{aligned}
$$

Likewise, it is shown in [5] that the following is a short exact sequence:

$$
0 \rightarrow H_{2}\left(X_{k}^{b}, X_{k}^{u}\right) \stackrel{\partial}{\rightarrow} H_{1}\left(X_{k}^{u}\right) \rightarrow H_{1}\left(X_{k}^{b}\right) \rightarrow 0 \text {. }
$$

Hence $\operatorname{rank}_{C} H_{1}\left(X_{k}^{b}\right)=\operatorname{rank}_{C}\left(X_{k}^{u}\right)-\mu=k(\mu-1)+c_{1}+1-\mu=(k-1)(\mu-1)+c_{1}$. Otherwise, $H_{q}\left(X_{k}^{b}\right) \cong H_{q}\left(X_{k}^{u}\right), 2 \leq q \leq n$.

The previous analysis took place over $C$, and the algebraic fact that all the polynomial invariants factored into linear factors in $\Psi$ considerably simplified the calculation. It is possible to make a similar analysis in $\Gamma$, which yields considerably more information in the case of prime power coverings. As before, $\bar{\beta}_{q}$ is the $q$-dimensional betti number of $X_{k}^{b}$.

Theorem 3. $n \geq 2$ or $n=1, \mu=1$ and $k=p^{r}, p$ a prime. Then

$$
\begin{aligned}
\bar{\beta}_{q} & =(k-1)(\mu-1), & & q=1, n+1, \\
& =1, & & q=0, n+2, \\
& =0, & & \text { otherwise. }
\end{aligned}
$$

Proof. Following the proof of Theorem 1, the analysis over $\Gamma$ boils down to looking at $\left(t^{k}-1\right)$ restricted to a cyclic summand $\Gamma / \lambda$. For the prime number $p$, we have the prime factorization in $\Gamma$ :

$$
\left(t^{p}-1\right)=(t-1)\left(t^{p-1}+t^{p-2}+\cdots+1\right)
$$

where the second factor is the pth cyclotomic polynomial $\phi_{p}(t)$. Moreover, we have the prime factorization $\left[6\right.$, p. 115] $\left(t^{p^{r}}\right)=(t-1) \phi_{p}(t) \phi_{p^{2}}(t) \cdots \phi_{p^{r}}{ }^{r}(t)$ 
where $\phi_{p_{v}^{v}}(t)=1+t^{p^{v-1}}+t^{2 p^{v-1}}+\cdots+t^{(p-1) p^{v-1}}$. Hence for $v \geq 1$ we have $\phi_{p} v^{v}(1) \geq 2$. Now the rational polynomial invariants $\left\{\lambda_{i}^{q}(t)\right\}, 1 \leq q \leq n$, have the property [5, Theorem 2.4] that $\lambda_{i}^{q}(1)= \pm 1$. This means that $\lambda_{i}^{q}(t)$ and $\left(t^{p^{r}}-1\right)$ are relatively prime, so the contribution to $H_{q}\left(X_{k}^{u} ; Q\right)$ from the $\Gamma$-torsion part of $H_{q}(\tilde{X} ; Q)$ is trivial for $q \geq 1$.

We are left with the case of a classical link, $n=1, \mu \geq 2$. In this case, we have $H_{2}(\widetilde{X} ; Q)=r_{2} \Gamma$, and

$$
H_{1}(\tilde{X} ; Q)=r_{1} \Gamma \oplus \Gamma / \lambda_{1}^{1} \oplus \cdots \oplus \Gamma / \lambda_{m_{1}}^{1} .
$$

Let $\xi$ be the greatest integer such that $\lambda_{\xi}^{1}(1)=0$. If $\lambda_{1}^{1}(1) \neq 0$, take $\xi=0$.

Theorem 4. $n=1, \mu \geq 2$. If $\bar{\beta}_{q}$ is the q-dimensional betti number of $X_{k}^{b}$, then $\bar{\beta}_{1}=\bar{\beta}_{2}=(k-1)(\mu-1)+c_{1}-k \xi$.

Proof. $\bar{\beta}_{1}=\bar{\beta}_{2}$ by Poincare duality, so it will suffice to compute $\bar{\beta}_{2}$. As in [4], [5] we have the short exact sequence of chain complexes

$$
0 \rightarrow C_{*}(\tilde{X} ; Q) \stackrel{(t-1)}{\longrightarrow} C_{*}(\tilde{X} ; Q) \rightarrow C_{*}(X ; Q) \rightarrow 0
$$

which induces the long exact sequence of homology

$$
0 \rightarrow H_{2}(\tilde{X} ; Q) \stackrel{(t-1)}{\longrightarrow} H_{2}(\tilde{X} ; Q) \rightarrow H_{2}(X ; Q) \rightarrow H_{1}(\tilde{X} ; Q) \rightarrow \cdots
$$

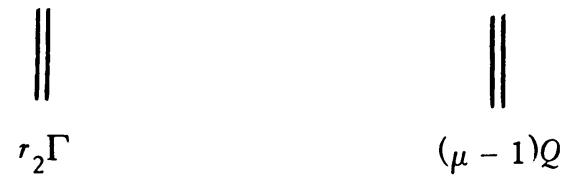

If $X$ fibers over $S^{1}$, then (3) is the Wang homology sequence of the fibration. From (3) we obtain the short exact sequence

$$
0 \rightarrow r_{2} Q \rightarrow(\mu-1) Q \rightarrow \xi Q \rightarrow 0 \text { so } r_{2}=(\mu-1)-\xi .
$$

Hence in the notation used in the proof of Theorem 1, we have

$$
\operatorname{Cok}_{2}=k r_{2} Q=k[(\mu-1)-\xi] Q, \quad \text { and } \quad \operatorname{Ker}_{1}=c_{1} Q \text {. }
$$

So

$$
\operatorname{rank}_{Q}\left(H_{2}\left(X_{k}^{u} ; Q\right)\right)=k(\mu-1)-k \xi+c_{1}
$$

and

$$
\operatorname{rank}_{Q}\left(H_{2}\left(X_{k}^{b} ; Q\right)\right)=\operatorname{rank}_{Q}\left(H_{2}\left(X_{k}^{u} ; Q\right)\right)-(\mu-1)=(k-1)(\mu-1)+c_{1}-k \xi .
$$


This is the same as the result obtained in [3, Theorem 2], except that the present version gives an explicit calculation for $\bar{\beta}_{1}$ in terms of the $\Gamma$-torsion invariants.

As in Theorem 3, by considering prime-power coverings we obtain more information. The following corollary generalizes the corresponding result found by Durfee (see [1]) for 2-fold branched cyclic coverings of algebraic links, and in it we adopt the hypotheses and notation of Theorem 4:

Corollary 5. $n=1, u \geq 2$ and $k=p^{r}, p$ a prime. Then $\bar{\beta}_{1} \leq(k-1)(\mu-1)$.

Proof. By the methods of [5, Theorem 2.4] we have

$$
\begin{aligned}
\lambda_{i}^{1}(1) & =0, & & i \leq \xi, \\
& = \pm 1, & & i \geq \xi+1 .
\end{aligned}
$$

Hence for $k$ a prime power, this means that $\left(t^{k}-1\right)$ and $\lambda_{j}^{1}(t)$ are relatively prime in $\Gamma$ in the range $j \geq(\xi+1)$. So the contribution to $c_{1}$ can come only from the $\lambda_{j}^{1}(t)$ with $j \leq \xi$, hence $c_{1} \leq k \xi$.

\section{REFERENCES}

1. A. H. Durfee, Branched cyclic covers and the characteristic polynomial of the local monodromy of plane algebraic curves (preprint).

2. L. Goeritz, Die Betti'schen Zählen der zyklischen Überlagerungsräume der Knotenaus enräume, Amer. J. Math. 56 (1934), 194-198.

3. F. Hosokawa and S. Kinoshita, On the homology group of branched cyclic covering spaces of links, Osaka Math. J. 12 (1960), 331-355. MR 23 \#A2878.

4. J. Milnor, Infinite cyclic coverings, Conference on Topology of Manifolds (Michigan State Univ., E. Lansing, Mich., 1967), Prindle, Weber \& Schmidt, Boston, Mass., 1968, pp. 115-133. MR 39 \#3497.

5. Y. Shinohara and D. W. Sumners, Homology invariants of cyclic coverings with application to links, Trans. Amer. Math. Soc. 163 (1971), 101-121. MR 44 \#2223.

6. B. L. van der Waerden, Moderne algebra. Vol. I, Springer, Berlin, 1930; English transl., Ungar, New York, 1949. MR 10, 587.

7. J. M. Woods, Some criteria for finite and infinite monodromy of plane algebraic curves, Invent. Math. (to appear).

8. O. Zariski, On the topology of algebroid singularities, Amer. J. Math. 54 (1932), 453-465.

DEPARTMENT OF MATHEMATICS, FLORIDA STATE UNIVERSITY, TALLAHASSEE, FLORIDA 32306

Current address: Institute for Advanced Study, Princeton, New Jersey 08540 